\title{
Dynamic compression of water to conditions in ice giant interiors
}

\author{
A. E. Gleason ${ }^{1,2 \square}$, D. R. Rittman ${ }^{2}$, C. A. Bolme ${ }^{3}$, E. Galtier ${ }^{4}$, H. J. Lee ${ }^{4}$, E. Granados ${ }^{4}$, S. Ali ${ }^{5}$, \\ A. Lazicki ${ }^{5}$, D. Swift ${ }^{5}$, P. Celliers ${ }^{5}$, B. Militzer ${ }^{6}$, S. Stanley ${ }^{7,8} \&$ W. L. Mao ${ }^{2}$
}

Recent discoveries of water-rich Neptune-like exoplanets require a more detailed understanding of the phase diagram of $\mathrm{H}_{2} \mathrm{O}$ at pressure-temperature conditions relevant to their planetary interiors. The unusual non-dipolar magnetic fields of ice giant planets, produced by convecting liquid ionic water, are influenced by exotic high-pressure states of $\mathrm{H}_{2} \mathrm{O}$-yet the structure of ice in this state is challenging to determine experimentally. Here we present $\mathrm{X}$-ray diffraction evidence of a body-centered cubic (BCC) structured $\mathrm{H}_{2} \mathrm{O}$ ice at $200 \mathrm{GPa}$ and $\sim 5000 \mathrm{~K}$, deemed ice XIX, using the X-ray Free Electron Laser of the Linac Coherent Light Source to probe the structure of the oxygen sub-lattice during dynamic compression. Although several cubic or orthorhombic structures have been predicted to be the stable structure at these conditions, we show this BCC ice phase is stable to multi-Mbar pressures and temperatures near the melt boundary. This suggests variable and increased electrical conductivity to greater depths in ice giant planets that may promote the generation of multipolar magnetic fields.

Understanding the phase diagram of $\mathrm{H}_{2} \mathrm{O}$, a ubiquitous molecule in the Universe and a primary building block of volatile-rich giant planets, is of crucial importance for condensed matter physics, solid-state chemistry, and planetary science. During the formation of Uranus and Neptune in the outer region of our solar system, massive amounts of $\mathrm{H}_{2} \mathrm{O}$ were accreted and are now stored at hundreds of GPa pressures in their interiors ${ }^{1}$. During the visit of the Voyager II spacecraft, its magnetometer revealed surprising non-axisymmetric, non-dipolar magnetic fields for the ice giants that differed substantially from the strong dipolar fields of Jupiter and Saturn ${ }^{2}$. Stanley and Bloxham ${ }^{3,4}$ performed numerical dynamo simulations using model geometries to explain Uranus' and Neptune's anomalous fields-finding their non-dipolar, non-symmetric magnetic fields are generated by a combination of electromagnetic stresses perturbing the convecting ionic fluid which surrounds a layered, stratified interior. Knowing the phases and properties of $\mathrm{H}_{2} \mathrm{O}$ at the pressure-temperature $(P-T)$ conditions of ice giant interiors on their isentropes is critical for validating dynamo simulations-but they are not well understood.

Convection of electrically conducting fluids generates magnetic fields in planetary interiors. If dissociation of molecules occurs in water-rich planets, then total conductivity is comprised of an electronic and ionic contribution. Ionic conduction is caused by the movement of negatively or positively charged ions and in the case of high-pressure $\mathrm{H}_{2} \mathrm{O}$ ice, protonic conductivity properties are crucial to constrain planetary dynamo processes (e.g., Refs. ${ }^{5,6}$ ). The existence of a proton fluid and an oxygen sub-lattice in the superionic phase raises questions about the response of this phase to electromagnetic stress through protonic fluid motion. Theoretical work has suggested a body-centered cubic (BCC) $)^{7,8}$, face-centered cubic (FCC) ${ }^{9-11}$, or orthorhombic (e.g., Ref. ${ }^{12}$ ) structure of $\mathrm{H}_{2} \mathrm{O}$ is stable at hundreds of GPa pressures and several thousand Kelvin, with bonding and transport properties consistent with a superionic phase.

More recent work ${ }^{13,14}$ use optical reflectivity, absorption measurements, and X-ray diffraction (XRD) to demonstrate the low electronic conductivity of ice and provide experimental evidence for superionic conduction of water ice in an FCC crystal structure, stable at pressure $(P)$, temperature $(T)$ conditions of $\sim 160 \mathrm{GPa}$ and calculated $3000 \mathrm{~K}$. The insulating solid ice phase at comparable pressure and below $2000 \mathrm{~K}$, ice X, is known to have a BCC lattice structure, but does not have superionic properties. In contrast, we find the first XRD evidence for a BCC structure between 100-200 GPa using compression-based solidification of liquid water calculated temperatures up to $\sim 5000 \mathrm{~K}$. This BCC crystal structure phase at these $P-T$ conditions represents a new phase

\footnotetext{
${ }^{1}$ Fundamental Physics Directorate, SLAC National Accelerator Laboratory, Menlo Park, CA 94025, USA. ${ }^{2}$ Geological Sciences, Stanford University, Stanford, CA 94305, USA. ${ }^{3}$ Shock and Detonation Physics, Los Alamos National Laboratory, Los Alamos, NM 87545, USA. 'Linac Coherent Light Source, SLAC National Accelerator Laboratory, Menlo Park, CA 94025, USA. 5Shock Physics, Lawrence Livermore National Laboratory, Livermore, CA 94550, USA. ${ }^{6}$ Earth and Planetary Science, University of California, Berkeley, CA 94720, USA. ${ }^{7}$ Earth and Planetary Sciences, Johns Hopkins University, Baltimore, MD 21218, USA. ${ }^{8}$ Applied Physics Lab, Johns Hopkins University, Laurel, MD 20723, USA. ${ }^{\circledR}$ email: ariannag@stanford.edu
} 


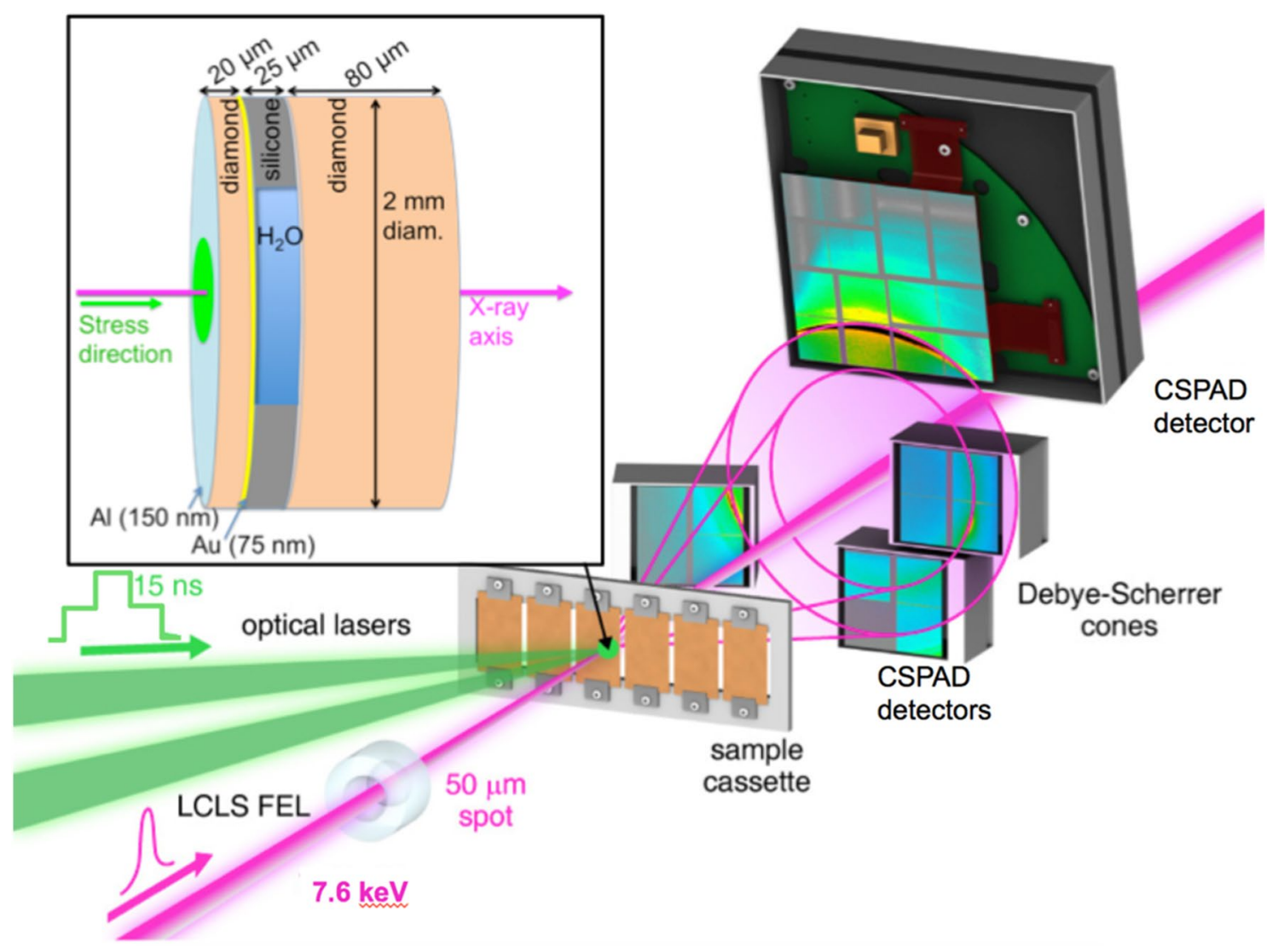

Figure 1. Experimental configuration of the XFEL probe and optical laser pump. The shock solidification behavior of water is captured in a Debye-Scherrer geometry. Inset: Schematic of target package with cut away side-view of the water layer.

of ice: ice XIX. Our constraints on the phase diagram of water ice near the conditions of the isentropes of ice giants, like Neptune and Uranus, have implications for their dynamos generating magnetic fields.

\section{Results}

Simultaneous, in situ XRD and velocimetry data combined with post-shot simulation work was used to examine the lattice structure, pressure, and temperature of $\mathrm{H}_{2} \mathrm{O}$. Here, atomic structure measurements of compressed liquid water $\left(\rho_{0}=1.0 \mathrm{~g} / \mathrm{cc} ; T_{0}=288 \mathrm{~K}\right)$ were made using transmission in situ XRD with $7.6 \mathrm{keV}$ X-rays from the $\mathrm{X}$-ray Free Electron Laser (XFEL) at the Matter in Extreme Conditions (MEC) end-station of the Linac Coherent Light Source (LCLS), SLAC National Accelerator Laboratory (Fig. 1). The applied loading scheme was reverberation compression-achieved through a temporally step-shaped drive laser (see "Methods"). The peak pressure was varied by changing the total number of Joules delivered to the target with a waveplate optic for the long pulse laser. The target geometry consisted of a clamp-style water containment approach [15). Individual packages of sandwiched diamond-water-diamond served as the targets: [20 $\mu \mathrm{m}$ thick chemical vapor deposited (CVD) diamond ablator $]+[25 \mu \mathrm{m}$ deionized water $(18 \mathrm{MOhm}$ ) layer set by a circular silicone washer (Silastic J, Dow Corning $)]+[80 \mu \mathrm{m}$ CVD diamond window $]$. Due to the impedance mismatch between the diamond ablator and the water, there is a many step compression sequence as elastic and plastic waves followed by reflections of those waves at interfaces effectively 'ringing' up the pressure in the water layer. A $75 \mathrm{~nm}$ gold layer was coated on the diamond 'ablator' surface in contact with the water sample, serving as the reflective layer for velocimetry and as an internal pressure calibrant to monitor compression via peak shifts in the XRD. The velocimetry data were recorded on a Velocity Interferometer System for Any Reflector (VISAR) diagnostic, simultaneously with the XRD (see "Methods") to provide an additional pressure constraint. The diamond 'window' served as containment for the water and was optically transparent to the VISAR probe allowing velocimetry measurements recording the motion from the Au.

Diffraction data, recorded on Cornell-SLAC Pixel Array Detectors (CSPADs), are azimuthally integrated (Fig. 2) as a function of $d$-spacing $(\AA)$ (see "Methods"). A representative trace of an integrated XRD pattern at ambient conditions shows strong intensity (111), (200) and (220) Au peaks plus CVD diamond peaks (from both the ablator and window). XRD records the reverberation-compressed sample at an XFEL probe timed to capture the diffraction after peak compression was achieved ( 7-9 ns). Polycrystalline diffraction peak positions are determined from peak fitting using Fityk ${ }^{16}$; Table 1 for a listing of run numbers, XRD peak $d$-spacings, hkl assignments, lattice parameters, densities, and estimated $P-T$ conditions. 


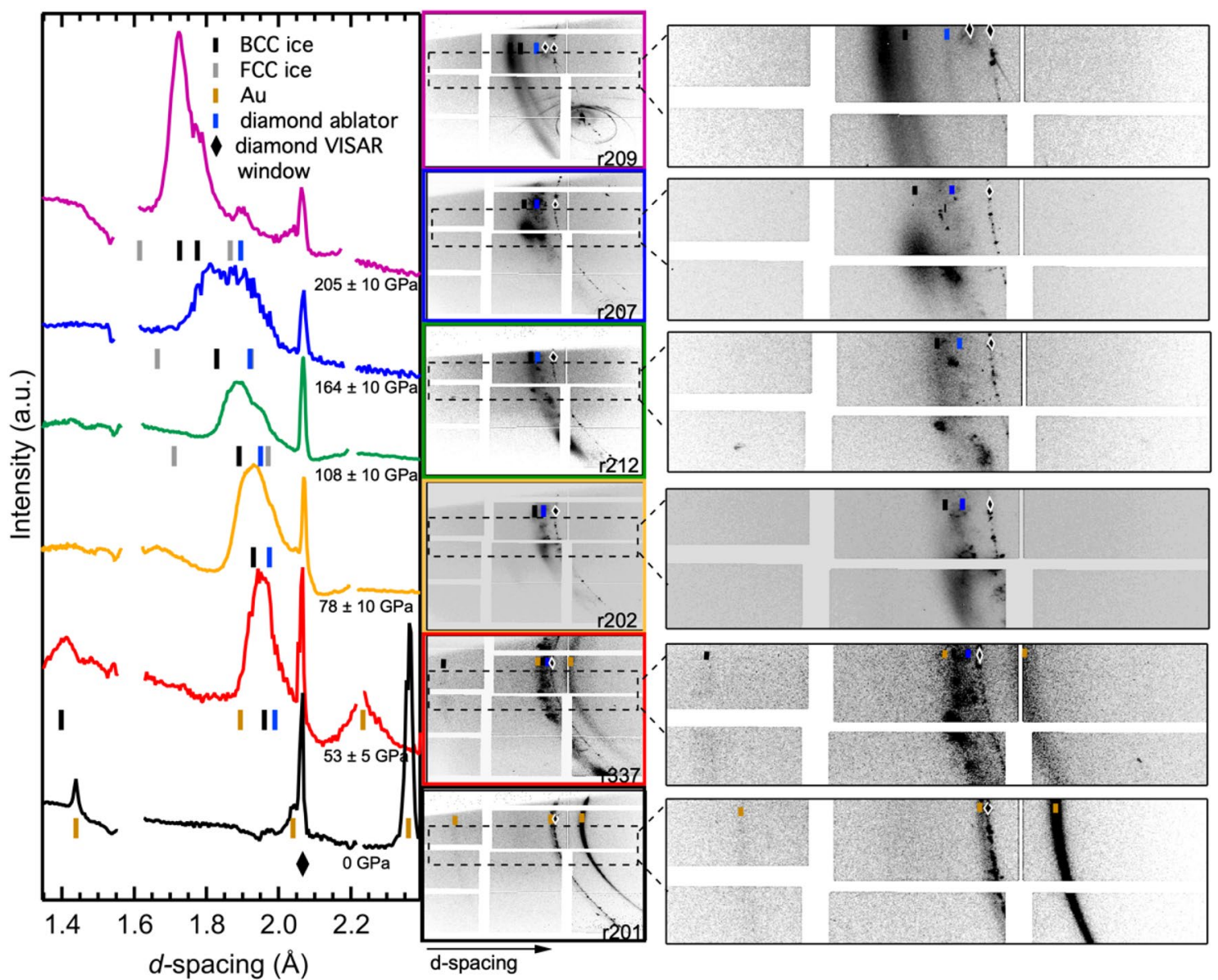

Figure 2. X-ray diffraction data with increasing pressure. Raw integrated traces from all high-pressure water shots without any normalization measured at $\sim 7 \mathrm{~ns}$ for the highest pressures. Colored tick marks indicate fitted peak center for labeled phase. FCC ice positions are shown for reference to note where peaks would be predicted to appear, but are not observed, at these pressures. The sharp peak at $2.07 \AA$ is the diamond peak (111) at near ambient conditions, labeled (filled diamond) from the VISAR-side window. $r 337$ peak assignment is particularly complicated due to superposed peaks from diamonds and ice. However, due to the presence of the (200) ice peak near 1.4 Å we can constrain the ice (110) to be a component of the diffraction intensity seen at $\sim 1.95 \AA$. Breaks between the detector pads are seen at $d$-spacings of 1.6 and $2.2 \AA$. Examples of 2 -dimensional raw CSPAD images with colored ticks to match phases listed in traces are shown to the right.

Diffraction from the (111) peak of the downstream diamond window shows little or no shift from the ambient $d$-spacing of $2.063 \AA$, indicating that the majority of the window volume is uncompressed. The lowest pressure XRD pattern records the Au (111) and (220) peaks shift to smaller $d$-spacing and broadening - providing a thermally corrected pressure of $47 \pm 3 \mathrm{GPa}, 1150 \mathrm{~K}\left(\right.$ Ref. $\left.^{17,18}\right)$; Fig. 2, red trace. The ablator CVD (111) diamond peak is also resolvable and shifts, consistent with $53 \pm 5 \mathrm{GPa}$ compression ${ }^{18-20}$. Two new XRD features are seen at 1.961(3) $\AA$ and 1.397(4) $\AA$ corresponding to the BCC ice VII structure, for (110) and (200), respectively, with a density of $2.78 \mathrm{~g} / \mathrm{cc}$, as expected in this regime $\mathrm{e}^{21}$. Within uncertainty, the densities of $\mathrm{Au}$, diamond, and ice are all consistent with a pressure of $53 \pm 5 \mathrm{GPa}$, also in agreement with the VISAR measurement of $56 \pm 4 \mathrm{GPa}$. Using previously published reverberation-compression-based equations of state (EoS) for ice $\mathrm{VII}^{7,19-25}$, we estimate a temperature of $1150 \pm 250 \mathrm{~K}$ for this density.

Upon increasing compression, the Au peaks are no longer resolvable in the XRD due to possible drive light leakage generating thermal expansion peak broadening. Although we clearly see Au peaks in the ambient patterns, above $\sim 50 \mathrm{GPa}$ we can no longer resolve the Au. Loss of clarity in the XRD data is likely due to peak broadening to the point that the diffuse scatter intensity distribution across the CSPAD prevents it from being resolved as discrete peaks. Possible reasons for broadening include: (1) reaching temperatures above the melting point of $\mathrm{Au}$ at these pressures, and/or (2) thermal expansion due to drive light leakage through the diamond ablator reaching Au layer before the compression process can take place. It has been documented that the drive laser spatial profile can spill over the chamfered drive side of the target mount and damaging neighboring targets. Due to laser light from a preceding shot reaching an adjacent target, the Al flash coating (150 $\mathrm{nm}$ ) on the drive side of the neighbor target can be damaged. The purpose of an Al flash coating is to prevent drive light from leaking through the ablator. However, if that $\mathrm{Al}$ coating was damaged, drive light can reach the Au layer, resulting in premature thermal expansion such that we cannot resolve the peaks. 


\begin{tabular}{|c|c|c|c|c|c|c|c|c|c|c|}
\hline Run & Phase & hkl & $d$-spacing $(\AA)$ & $a(\AA)$ & $\mathbf{V}\left(\AA^{3}\right)$ & $\rho(g / c c)$ & $\mathrm{Up}(\mathrm{km} / \mathrm{s})$ & P_visar (GPa) & P_xrd (GPa) & Average P (GPa), T (K) \\
\hline \multicolumn{11}{|l|}{201} \\
\hline & $\mathrm{Au}^{*}$ & 111 & $2.361(1)$ & 4.079 & 67.87 & 19.28 & & & 0 & \\
\hline & & 200 & $2.043(3)$ & 4.079 & 67.87 & 19.28 & & & 0 & \\
\hline & & 220 & $1.437(1)$ & 4.079 & 67.87 & 19.28 & & & 0 & \\
\hline & diamond- $\mathrm{V}, \mathrm{ab}$ & 111 & $2.063(1)$ & 3.574 & 45.63 & 3.49 & 0 & 0 & 0 & 0,300 \\
\hline \multicolumn{11}{|l|}{337} \\
\hline & $\mathrm{Au}^{*}$ & 111 & $2.233(3)$ & 3.868 & 57.86 & 22.61 & & & 47.0 & \\
\hline & diamond-V & 111 & $2.063(3)$ & 3.574 & 45.63 & 3.49 & & & & \\
\hline & diamond-ab^ & 111 & $1.989(4)$ & 3.445 & 40.89 & 3.90 & $1.2(1)$ & 57.0 & 53.0 & \\
\hline & ice_bcc VII & 110 & $1.961(3)$ & 2.782 & 21.53 & 2.78 & & & & $53(5), 1150(250)$ \\
\hline & ice_bcc VII & 200 & $1.397(4)$ & 2.782 & 21.53 & 2.78 & & & & $53(5), 1150(250)$ \\
\hline \multicolumn{11}{|l|}{202} \\
\hline & diamond-V & 111 & $2.069(5)$ & 3.574 & 45.63 & 3.49 & & & & \\
\hline & diamond-ab^ & 111 & $1.975(3)$ & 3.420 & 40.00 & 3.99 & $1.7(1)$ & 84.6 & 78.1 & \\
\hline & ice_bcc X & 110 & $1.930(5)$ & 2.730 & 20.35 & 2.94 & & & & $78(10), 1800(350)$ \\
\hline \multicolumn{11}{|l|}{212} \\
\hline & diamond-V & 111 & $2.067(3)$ & 3.574 & 45.63 & 3.49 & & & & \\
\hline & diamond-ab^ & 111 & $1.949(5)$ & 3.377 & 38.51 & 4.14 & $2.3(2)$ & 114.9 & 107.8 & \\
\hline & ice_bcc XIX & 110 & $1.889(2)$ & 2.680 & 19.25 & 3.11 & & & & $108(10), 2200(350)$ \\
\hline \multicolumn{11}{|l|}{207} \\
\hline & diamond-V & 111 & $2.071(3)$ & 3.574 & 45.63 & 3.49 & & & & \\
\hline & diamond-ab^ & 111 & $1.920(7)$ & 3.326 & 36.79 & 4.34 & $3.2(3)$ & 171.7 & 164.0 & \\
\hline & ice_bcc XIX & 110 & $1.828(6)$ & 2.587 & 17.31 & 3.46 & & & & 164(10), 2700(500) \\
\hline \multicolumn{11}{|l|}{209} \\
\hline & diamond-V & 111 & $2.064(3)$ & 3.574 & 45.63 & 3.49 & & & & \\
\hline & diamond-ab^ & 111 & $1.895(3)$ & 3.282 & 35.35 & 4.51 & $4.5(4)$ & 245.5 & 205.4 & \\
\hline & ice_bcc XIX & 110 & $1.770(3)$ & 2.504 & 15.70 & 3.81 & & & & $205(10), 5500(500)$ \\
\hline & ice_bcc XIX & 110 & $1.725(3)$ & 2.440 & 14.53 & 4.12 & & & & $205(10), 3300(500)$ \\
\hline \multicolumn{11}{|l|}{399} \\
\hline & diamond-V & 111 & $2.069(3)$ & 3.574 & 45.63 & 3.49 & & & & \\
\hline & diamond-ab^ & 111 & $1.894(4)$ & 3.281 & 35.32 & 4.52 & $4.5(5)$ & 245.5 & 207.5 & \\
\hline & ice_bcc XIX & 110 & $1.769(5)$ & 2.502 & 15.66 & 3.82 & & & & $207(10), 5500(500)$ \\
\hline & ice_bcc XIX & 110 & $1.724(4)$ & 2.438 & 14.49 & 4.13 & & & & $207(10), 3300(500)$ \\
\hline
\end{tabular}

Table 1. Peak assignment and lattice parameters. $V$ VISAR-side diamond, $a b$ ablator-side diamond. ${ }^{\star}$ Pressure and temperature determined using Fei et al..$^{18}$ and $\mathrm{Marsh}^{19}{ }^{\wedge} \wedge$ Pressure determined using P-rho of McWilliams et al. ${ }^{20}$; Knudson et al. ${ }^{21}$ and Marsh ${ }^{19}$ and LEOS9061 $1^{30}$.

Peak shifts in the compressed diamond ablator are resolvable and, using these diffraction peaks as a pressure calibrant in combination with the velocimetry record, we track pressure increasing to just over $\sim 200 \mathrm{GPa}^{18-20}$. The reported pressures are determined from the XRD of the diamond-ablator and are corroborated, within the uncertainty, by the pressure determined from the velocimetry traces. Pressure uncertainty is taken from the goodness-of-fit value for a Gaussian peak profile of the ablator diamond diffraction peak $d$-spacing, converted to a density uncertainty and used to estimate the and pressure uncertainty with an equation of state. We see the water ice diffraction peak shift from 1.961(3) $\AA$ (at $53 \mathrm{GPa}$ ) to 1.725(3) $\AA$ at the highest pressure. If we continue to assign this feature as a BCC (110) peak, the ice pressure estimates (via EoS from Refs. ${ }^{7,19-21}$ ) track well with the compressed diamond ablator estimates up to $\sim 160 \mathrm{GPa}$. Beyond this pressure there are discrepancies in the EoS results between quantum molecular dynamics simulations (e.g., Ref. ${ }^{24}$ ) and previous experiments (e.g., Refs. ${ }^{21,22,26}$ ) for water. At the highest pressure, $205 \pm 10 \mathrm{GPa}$, indexing the new peak as a BCC (110) gives a lattice parameter of $a=2.440 \AA$, corresponding to a density of $4.12 \mathrm{~g} / \mathrm{cc}$. Unfortunately, the (200) peak for the BCC ice structure falls off the detector $d$-spacing range above $\sim 75 \mathrm{GPa}$.

Above the ice VII and ice X P-T stability fields, we can test the viability of the FCC, hexagonally-close packed (HCP), and orthorhombic structures, assuming the geometric constraints of packing efficiency or close packing oxygen in three dimensions (e.g., Refs. ${ }^{10,12}$ ). Our procedure was to test assignment of the new peak visible in pressures above $150 \mathrm{GPa}$ to an FCC, HCP, or orthorhombic $(\mathrm{Pbcm})$ structure and then inspect the 2-dimensional CSPAD images for any diffraction intensity located near a predicted $(\mathrm{hkl}) d$-spacing position for that structure. If we assign the FCC (111) peak to the $1.828 \AA$ feature, the corresponding FCC (200) should be at $1.583 \AA$ which should then shift to $1.50 \AA$ with compression. We do not see any XRD signal at these positions on the CSPADs. Similarly, if the HCP structure were assigned at comparable ice densities, the (101) is missing at its predicted 


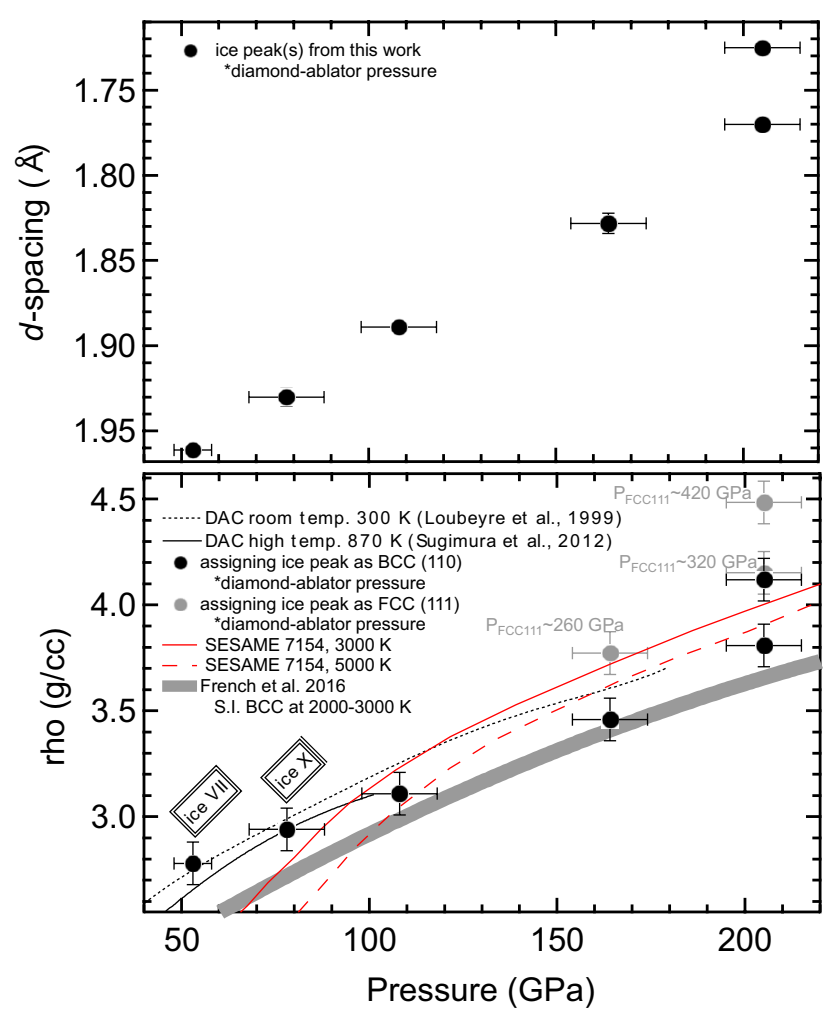

Figure 3. Comparison of $d$-spacing and density for different structures. Measured $d$-spacings for each ice peak plotted with the pressures derived from the diamond-ablator XRD signal (top graph). Black circles (bottom graph) show density trend with pressure assigning this peak as the BCC (110). Dotted black line is the Vinet fit to room temperature, static compression DAC work Loubeyre et al. ${ }^{40}$. Solid black line is the trend for high temperature DAC data Sugimura et al. ${ }^{35}$. We note a reasonable similarity with this trend and the DFT prediction from French et al. (Ref..$^{25}$; solid grey line) for a superionic BCC structure of ice below $200 \mathrm{GPa}$, but above there is some discrepancy. Red curves are from SESAME 7154 (Ref. ${ }^{26}$ ) for $3000 \mathrm{~K}$ and $5000 \mathrm{~K}$, solid and dashed, respectively. Assigning the same $d$-spacing peak to FCC (111) (grey circles) shows a marked jump in density using the diamond-ablator based pressure which does not fit any predicted trend, nor do we have the corresponding FCC (200) peak which would be in the detector range. If we use French et al. ${ }^{25}$ EoS for superionic FCC ice to determine pressure of the ice, there would be a marked jump in pressure which does not corroborate the diamond-ablator pressure, or velocimetry-based pressure or laser drive intensity-pressure calibration data.

$1.843 \AA$, or $1.678 \AA$, respectively for $\rho_{\mathrm{HCP}}=3$ and $4 \mathrm{~g} / \mathrm{cc}$. Regarding the orthorhombic $\mathrm{Pbcm}$ structure-we also check for peaks using a linear extrapolation of lattice parameters, e.g., Ref. ${ }^{12}$, to $\sim 200 \mathrm{GPa}$ to look for (110) and (101) peaks at $1.96 \AA$ and $1.47 \AA$, respectively. Figure 3 compares $d$-spacing vs pressure and corresponding densities at each pressure assuming a BCC or FCC structure, including previous diamond-anvil cell data. Due to the absence of any corresponding FCC, HCP, or Pbcm peaks, each expected to be within the detector $d$-spacing coverage with predicted relative intensities of these peaks well above the noise floor of the detectors, and a BCC ice density consistent with velocimetry-based pressure estimates and compressed diamond-ablator pressures estimates, we conclude that the ice structure seen in the XRD data is BCC. At similar shot conditions, the formation of this BCC structure is reproducible (Fig. 4).

Previous dynamic compression induced disorder-order transitions (e.g., Ref. ${ }^{27}$ ) have reported randomlyoriented nanocrystalline growth of the high pressure phase as seen in the uniformity of Debye-Scherrer ring intensity and the relative peak intensities matching a randomly orientated powder distribution. However, we see interesting trends in the change in powder XRD texture for both the ice phase and diamond above $100 \mathrm{GPa}$. The CVD ablator diamond signal remains spotty, showing a similarly sized grain structure as it compresses in the elastic regime up to the $\sim 80 \mathrm{GPa}$ HEL. Above this pressure, we see a gradual increase in ring smoothness and peak broadening up to the highest pressure of $\sim 200 \mathrm{GPa}$ where the intensity is more uniform over the azimuthal range available. This may indicate that the grain size is likely decreasing and orientations are becoming more random. The ice VII and ice X diffraction are large, broad and spotty up to over $100 \mathrm{GPa}$. Then as the ice $\mathrm{X}$ transitions to ice XIX above $\sim 150 \mathrm{GPa}$ we see these larger spots become more diffuse at the edges, perhaps indicating some increase mosaicity and/or crystallites with preferred orientation with respect to the compression direction. In the highest pressure shot there is an apparent concentration of diffraction intensity for the BCC ice (110) peak near the top of the detector. This is consistent with the horizontally polarized XFEL probe (considering the orientation of the CSPAD active areas with respect to the XFEL propagation direction). Additionally, we note heterogeneous growth of ice crystals on target component interfaces may have a needle-like geometry ${ }^{15,28}$, 


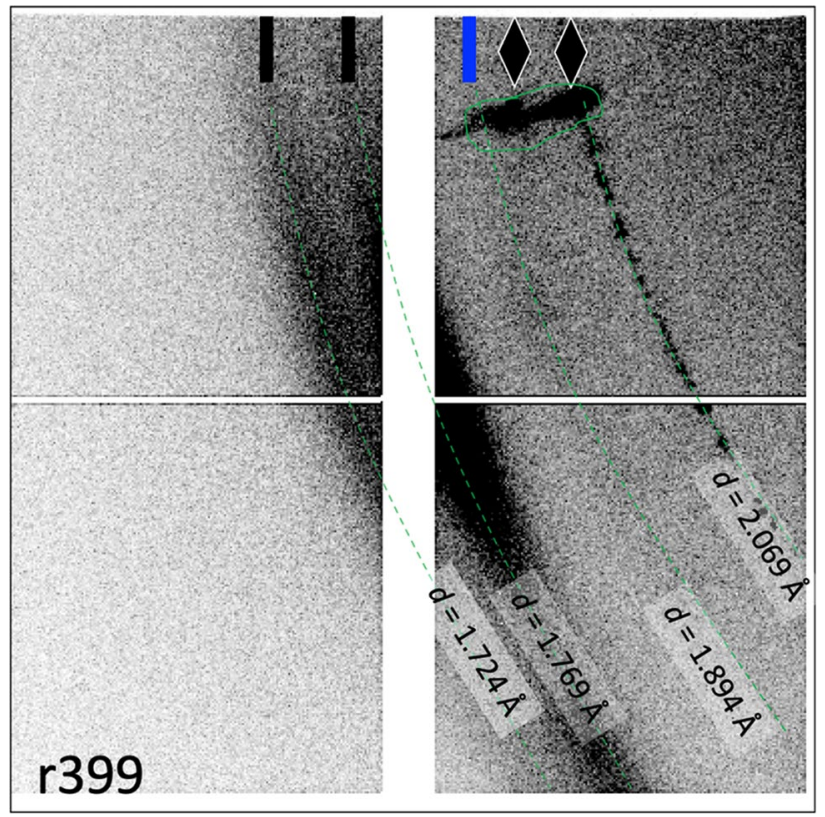

Figure 4. Raw data from Run 399. Repeat of conditions from Run 209, this Run 399 shows diffraction signal from ice XIX with the same doublet feature (black ticks, $d$-spacings $=1.724 \AA, 1.769 \AA$ ), the ablator diamond (blue tick, $d$-spacing $=1.894 \AA$ ), the VISAR window diamond (black diamonds, $d$-spacing $=2.069 \AA$ and elongated feature at the top of the image which could be due to a small volume fraction of the single crystal responding to the stress distribution from compressive wave interactions). Light green lines are guides for the eye or to outline features.

such that nanocrystallites develop preferential orientation with respect to the X-ray probe direction. This could also contribute to a concentration of diffraction intensity at this location on the detector.

Velocimetry data was obtained by analyzing the line VISAR interferograms measured in the experiments with an image reduction routine ${ }^{29}$, which employed a Fourier transform method to extract the interferograms' phase information. The spatially resolved velocity histories were acquired by applying the experimental velocity-perfringe to the extracted phase map of the data (representative VISAR, Fig. 5). The drive laser pulse used to achieve these conditions is shown in Fig. 6. Equations of state (EoS) from SESAME 7154 and LEOS 9061 were used for the water and diamond ablator, respectively ${ }^{26,30}$, finding these are comparable to those used in Millot et al. ${ }^{13,14}$. Due to the large impedance mismatch between the diamond and the water, the initial shock wave in diamond generates a release wave at the diamond-water interface which is reflected back into the diamond ablator. The impedance difference persists and sets up a reverberating shock in the diamond ablator. Breakout of the first shock in the ablator diamond into the water is at 1-2 ns (labeled 'b/o' for breakout, Fig. 5). The ablator diamond reverberation results in the diamond plastic wave overtaking the weak elastic wave in the water and reached the water-VISAR diamond interface at roughly $\sim 5 \mathrm{~ns}$. A 1 st and 2 nd shock wave transits the VISAR-side diamond to breakout into vacuum by $\sim 8 \mathrm{~ns}$ and $9.5 \mathrm{~ns}$.

The HYADES Radiation Hydrodynamics $\operatorname{code}^{31}$ was used to perform a post-shot simulation and model the wave propagation through the target package (Fig. 7). These simulations compare well with velocimetry data-confirming the timing of expected features, like breakout of the elastic wave from the ablator-diamond, the main pressure wave reaching the downstream water-diamond interface near $4.5 \mathrm{~ns}$, and shock waves breaking out into vacuum at around $\sim 8-9 \mathrm{~ns}$. Since we had no separate temperature diagnostic, we can only rely on this post-shot simulation temperature estimate and known $P-T$ EoS for water under reverberation compression condition $s^{21-23,26}$ to constrain our temperature. Post-shot simulations were completed for high- and low-pressure shots using the Hyades Radiation Hydrodynamics code. We found that these shots required a multiplier of 0.45 to obtain a best match to VISAR data. A typical multiplier value is $\sim 0.7$ (Ref. ${ }^{32}$ ), however our small multiplier value is indicative of extensive laser energy loss before reaching the target. This could be due to optics/coating damage in the beam path decreasing the delivered intensity reaching the target - perhaps up to $65 \%$.

Diffraction from the highest pressure shot shows a doublet feature for the BCC (110). The hydrodynamic simulation shows a bimodal temperature distribution at the $7 \mathrm{~ns}$ probe time, present in the ice in two discrete ice layers: at $\sim 3300 \mathrm{~K}$ and at $5500 \mathrm{~K}$. This $\sim 2000 \mathrm{~K}$ temperature difference between the layers would manifest as a density difference of $30-35 \%$ in the ice, which is consistent with the peak separation using the SESAME 7154 Gruneisen parameter $\left(\right.$ Ref. $\left.^{26}\right)$. Because of the uncertainty in pressure and temperature conditions for this shot we list out two data points for BCC, one for each peak of the doublet giving: $205 \mathrm{GPa}, 3300 \mathrm{~K}$ and $205 \mathrm{GPa}, 5500 \mathrm{~K}$. 


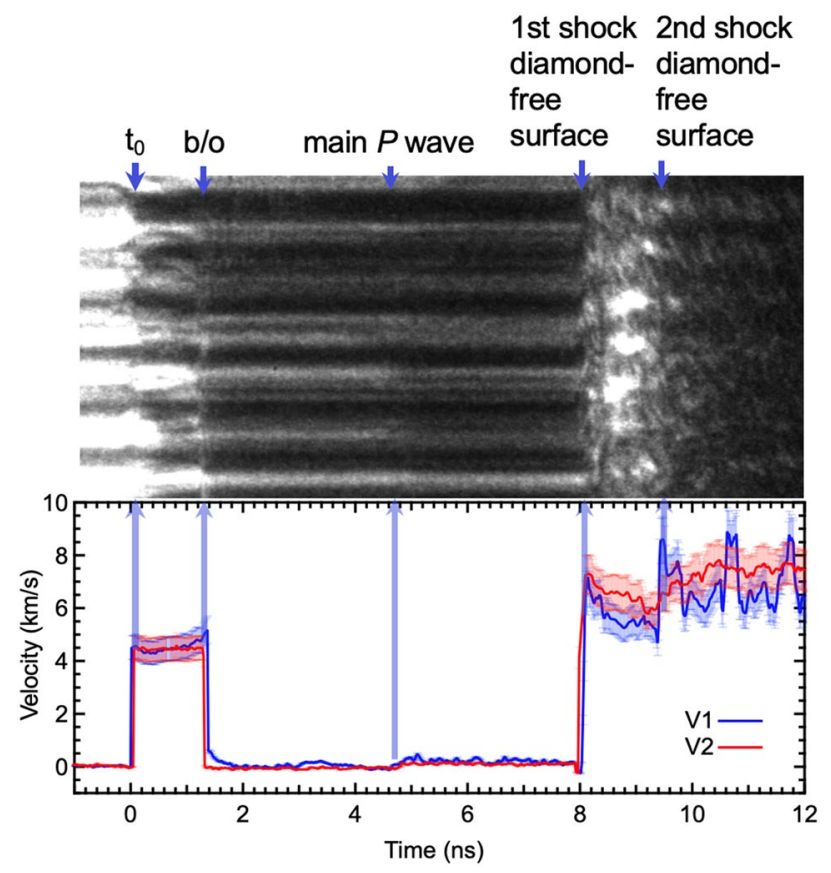

Figure 5. Velocimetry data from reverberation compression of water to $\sim 200 \mathrm{GPa}$. Example raw VISAR1 (blue line \& streak camera image) and VISAR2 (red line) data from Run 209 over a $28 \mu \mathrm{m}$ region showing particle velocity (Up) of the diamond ablator $\left(\sim 4.5 \mathrm{~km} \mathrm{~s}^{-1}\right)$ and free surface velocities (Ufs) from the 1st and 2nd shock arrivals at the diamond VISAR window $\left(\sim 7-7.5 \mathrm{~km} \mathrm{~s}^{-1}\right)$ ranging from 4.4 to $6 \mathrm{~km} \mathrm{~s}^{-1}$. Due to VISAR quality, the Up uncertainty is $\sim 10 \%$. Breakout (b/o) of the diamond elastic wave into the water layer occurs at $\sim 1.5 \mathrm{~ns}$, followed by the main pressure wave reaching the VISAR-side diamond-water interface at $\sim 4.5 \mathrm{~ns}$. The 1 st and 2nd shockwave arrivals reach the VISAR-side diamond-vacuum interface at $\sim 8 \mathrm{~ns}$ and $9.5 \mathrm{~ns}$, respectively. Drive laser parameters listed in Table 2.

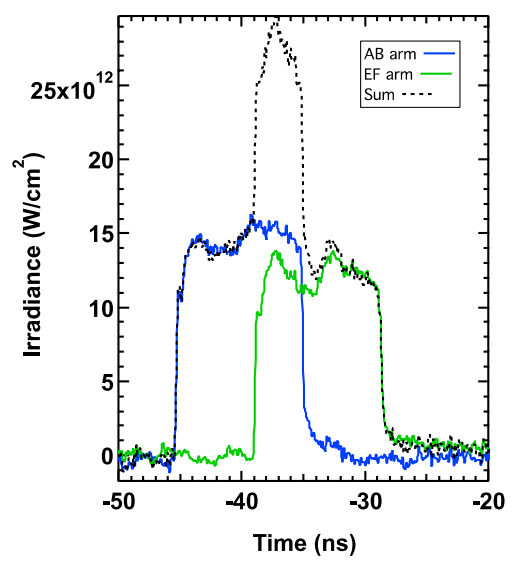

Figure 6. Oscilloscope traces of drive laser temporal profiles. Blue and green traces show the two 10 ns flat-top profiles offset by $\sim 5$ ns to yield a stepped $\sim 15$ ns drive pulse profile. Two separate laser 'arms' are combined to generate the compression wave, here labeled $\mathrm{AB}$ and $\mathrm{EF}$ corresponding to names of the capacitor banks. The total irradiance as seen by the target is the sum of the two drive profiles shown by the dotted black line.

\section{Discussion}

Direct observation of the crystal structure of $\mathrm{H}_{2} \mathrm{O}$ ice at a pressure of $\sim 200 \mathrm{GPa}$ and a calculated temperature of $5000 \mathrm{~K}$ has implications ranging from the fundamental physics and chemistry of $\mathrm{H}_{2} \mathrm{O}$ to ice giant dynamo evolution. XRD presented here provides the first evidence of a BCC ice structure at these conditions. Consistent with superionic behavior, water is predicted to have a band gap $(2-3 \mathrm{eV})$ at these conditions causing it to absorb visible light (e.g., Ref. ${ }^{\text {) }}$, and we do see the loss of reflectance of the $532 \mathrm{~nm}$ probe light off the Au as the shock front transits the $\mathrm{H}_{2} \mathrm{O}$ layer. However, since our velocimetry records are inconclusive regarding a direct transport property measurement, we also consider alternate explanations. For instance, reflectance loss at 2-8 ns (Fig. 5) 

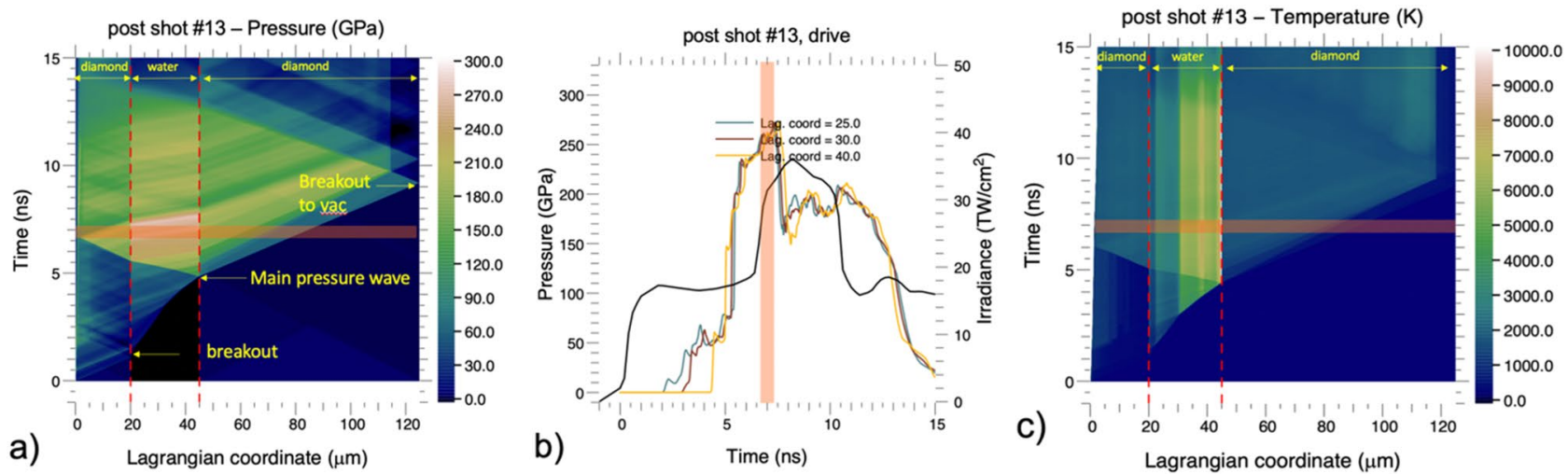

Figure 7. Lagrangian distance-time diagrams. A post-shot simulation for (a) pressure labeled by color code (b) pressure line outs of specific Lagrangian coordinates in the water layer and (c) temperature distribution. In (a) and (c), the red dashed line shows the water region. Solid black line in (b) shows drive profile. Probe time of the X-rays was at $6.95 \pm 0.35 \mathrm{~ns}$ (orange bar) where line width includes probe time uncertainty derived from rise time of first pulse $(0.15 \mathrm{~ns})$ and timing jitter of laser $(0.20 \mathrm{~ns})$.

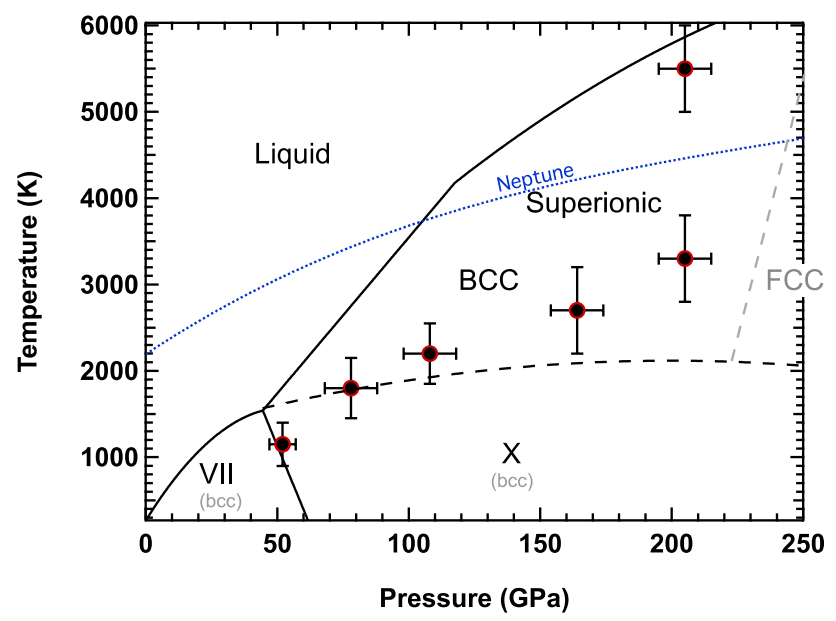

Figure 8. Phase diagram of $\mathrm{H}_{2} \mathrm{O}$. Revised phase diagram for $\mathrm{H}_{2} \mathrm{O}$ (adapted from Refs. ${ }^{9,10,14,25,40,41}$ including our data for BCC-structured ice (black circles) which shifts the FCC boundary to higher pressures in line with theoretical predictions from Refs. $\left.{ }^{10,25}\right)$. Blue dashed line is the Neptunian isentrope ${ }^{36}$.

could also result from the light scattering off BCC ice grain boundaries (or a combination of both phenomena). Using the Scherrer Equation ${ }^{33}: \phi=\frac{K \lambda}{\beta \cos \theta}$, where $\phi=$ grain size; $K=$ dimensionless shape factor (commonly set to 0.9 ); $\lambda=\mathrm{X}$-ray wavelength; $\beta=$ line broadening at full width at half maximum (FWHM) minus instrumental broadening $\left(0.03^{\circ}\right) ; \theta=$ Bragg angle, the BCC $(110)$ ice peak width gives a grain size of $21 \pm 2 \mathrm{~nm}$, similar to the findings of Millot et al. ${ }^{14}$. This small grain size could lead to scattering of the VISAR probe light, which could cause the apparent loss of Au reflectivity. We do note that previous experiments ${ }^{13,14,34,35}$ and computations (e.g., Ref. ${ }^{9,24,25}$ ) have shown that the $P-T$ conditions achieved here is within the superionic phase stability field. Applying our new XRD data to the phase diagram of high pressure ice confirms the BCC structure previously theorized is stable at these conditions (e.g., Refs. ${ }^{10,14,25}$ ) (Fig. 8) in the superionic regime. Recent pioneering work by Millot et al. ${ }^{13,14}$ has also examined the phase diagram of water, to pressures beyond this paper, under laserdriven shock-compression. We compare the lattice structure of ice at similar $P, T$ conditions and find evidence of a BCC ice structure near the liquid boundary suggesting the FCC phase stability region can be pushed out to higher pressure. Millot et al. ${ }^{14}$ find an FCC ice extending to the liquid boundary. Our data suggest pushing this FCC boundary out to higher pressures ( $>250 \mathrm{GPa})$ along the isentrope. However, this pressure assignment, based on diamond XRD could represent the lower bound for the ice pressure, and in fact, be at higher pressure as indicated by hydrocode assessment. Our results are consistent with all but one of the data points reported by Millot et al. ${ }^{14}$. The reason for this discrepancy is not yet understood, but could be related to issues in diffraction quality signal/noise and indicates the need for more experimental investigations working to resolve real-time diffraction for phase with higher Q-range, velocimetry for pressure, and pyrometry for temperature determination all collected with in situ diagnostics.

Due to the nature of packing of the oxygen sub-lattice, the BCC structure is generally thought to have a higher hydrogen mobility than the FCC structure. The BCC structure allows the hydrogen atoms to migrate 


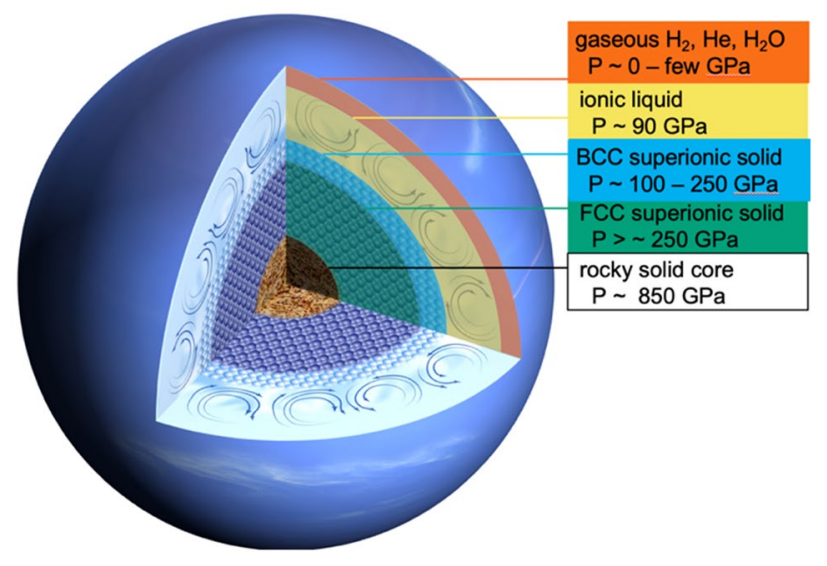

Figure 9. Neptune interior with multiple superionic layers. Ice giant interiors, like Neptune, have different layers of superionic ice. The molecular envelope of $\mathrm{He}, \mathrm{H}_{2}$ and $\mathrm{H}_{2} \mathrm{O}$ gas is largely insulating and the convecting ionic liquid layer may have ionic conductivities of a few to $100(\Omega \mathrm{cm})^{-1}$. However, the superionic solid BCC and FCC layers can have comparable protonic conductivity $100(\Omega \mathrm{cm})^{-1}$ or up to two orders of magnitude larger, depending on the estimated proton mobility (or diffusion coefficient $D_{H}=1.8 \mathrm{e}-3 \mathrm{~cm}^{2} \mathrm{~s}^{-1}$ (Refs. ${ }^{6,36}$ ) to $0.5 \mathrm{~cm}^{2} \mathrm{~s}^{-1}$ (Ref. ${ }^{9}$ ) at relevant pressures and temperatures along the isentrope.

freely between different, connected interstitial sites, i.e., tetrahedral or octahedral, whereas the FCC structure

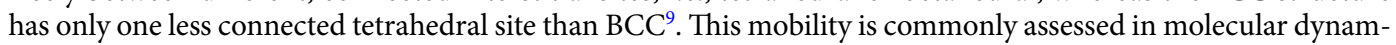
ics calculations as the hydrogen diffusion rate ${ }^{8,9,11,36}$. A linear extrapolation of the hydrogen diffusion constant with density to the conditions probed in this study suggests the BCC hydrogen diffusion constant is $40 \%$ higher than the FCC diffusion constant ${ }^{9}$. The values for the diffusion coefficient vary over many orders of magnitude, $1.8 \mathrm{e}-3 \mathrm{~cm}^{2} \mathrm{~s}^{-1}$ (Refs. ${ }^{11,36}$ ) to $\sim 0.5 \mathrm{~cm}^{2} \mathrm{~s}^{-1}$ (Ref. ${ }^{9}$ ) for pressures and temperatures measured in this study for a BCC structure, depending on the molecular dynamics simulation parameters. The relationship between hydrogen diffusion coefficient $\left(D_{H}\right)$ and protonic conductivity $(\sigma)$ is governed by the Nernst-Einstein equation ${ }^{37,38}: \sigma=\frac{f^{f n D_{H} q^{2}}}{R T}$, where $f$ is non-dimensional geometrical constant taken to be $1, n$ is the molar concentration per unit cell volume, $q$ is the charge, $R$ is the gas constant, and $T$ is the temperature, and shows a proportional relationship between hydrogen diffusion coefficient and protonic conductivity. For our BCC lattice parameter $(2.440 \AA)$, and a $D_{H}$ of $1.8 \mathrm{e}-3 \mathrm{~cm}^{2} \mathrm{~s}^{-1}\left(\right.$ Ref. $\left.^{36}\right)$ we find a protonic conductivity of $102(\Omega \mathrm{cm})^{-1}$, whereas an FCC structure at the same pressure would be $\sim 20 \%$ lower conductivity. This is in contrast to the liquid outer region of Neptune where ionic conductivity at 25 to $100 \mathrm{GPa}$, ranges from $<1$ to $30-90(\Omega \mathrm{cm})^{-1}$, respectively (e.g., Ref. $\left.{ }^{6,39}\right)$. Confirmation of the extension of the BCC high-pressure stability field in the superionic regime results in higher than predicted protonic conductivity to greater depths in ice giant interiors (Fig. 9) which can respond well to magnetic stress.

Ice giant dynamos are generated in a convecting, fluid layer of electrically conductive water, ammonia, and methane. Solid ice layers cannot participate in the dynamo action through fluid motions. However, dynamo complexities arising from layered superionic ices with high but differing proton mobilities will influence the magnetic field properties. Here we have shown that solid ice is stable to over $200 \mathrm{GPa}$ and $4400 \mathrm{~K}$ in a BCC structure. Combined with previous measurements that indicate that water ice is superionic in this $P-T$ region $^{13}$, this suggests that the lower boundary of the dynamo-generation region is likely related to the location of the superionic phase change, especially if that phase change results in a solid ice layer (e.g., Ref. ${ }^{6}$ ), as seen here. Our data change the sub-liquid layer from FCC superionic solid ice to BCC superionic solid ice, resulting in a $40 \%$ increase in protonic conductivity. The magnetic fields generated in the ionic liquid layer will interact differently with a BCC ice layer than they would an FCC ice layer due to this difference in conductivity. For example, the magnetic fields generated in the ionic fluid layer are time-varying and the skin depth of penetration of magnetic fields into any solid sub-layer depends on the conductivity of that solid material. A higher conductivity solid sub-layer would preferentially repel magnetic fields, limiting their length scales. Although dynamo simulations have been able to produce multipolar magnetic fields without an enhanced conductivity of a solid interior (e.g., Ref. $^{3}$ ), the smaller length-scales resulting from the enhanced repulsion of a higher conducting solid interior would promote the generation of multipolar magnetic fields-consistent with measurements made by Voyager II for Uranus and Neptune.

\section{Methods}

Experimental design. Quasi-monochromatic $(\mathrm{dE} / \mathrm{E}=0.2-0.5 \%)$, fully transverse coherent, 7.603(30) keV $\mathrm{X}$-ray pulses of $40 \mathrm{fs}$ duration with an average of $\sim 10^{12}$ photons per pulse, were incident over a $50 \mu \mathrm{m}$ diameter spot on the target package. An X-ray only shot was collected before the drive shot as a reference. The $50 \mu \mathrm{m}$ XFEL beam spot did not produce any observable $\mathrm{x}$-ray damage to the target. Metal coatings on the diamond ablator served to absorb the drive laser ( $150 \mathrm{~nm} \mathrm{Al}$ on upstream side) and act as the reflective layer for velocimetry measurements (75 $\mathrm{nm}$ Au on downstream side). 


\begin{tabular}{|c|c|c|c|c|c|}
\hline \multirow[b]{2}{*}{ Run } & \multicolumn{2}{|c|}{ Radius [cm] } & \multirow[b]{2}{*}{ Energy [J] } & \multirow[b]{2}{*}{ Duration [ns] } & \multirow[b]{2}{*}{ Spot size $\left[\mathrm{cm}^{2}\right]$} \\
\hline & r_a & r_b & & & \\
\hline r209AB & 0.0048 & 0.0063 & 15.3 & 10.2 & $9.50018 \mathrm{E}-05$ \\
\hline r209EF & 0.0053 & 0.0058 & 13.4 & 10.2 & $9.65726 \mathrm{E}-05$ \\
\hline
\end{tabular}

Table 2. Drive laser parameters. Drive laser spatial profile (radius) was made using an equivalent plane monitor at the target position and captured as a CCD tif image. ImageJ was use to extract the illumination profile and FWHM from this image. Energies are calibrated using a diode recording light leakage from a mirror in the laser enclosure. Pulse duration is recorded on an oscilloscope for each shot.

The optical drive laser was defocused to a $100 \mu \mathrm{m}$ diameter spot at FWHM with a Gaussian spatial profile to achieve focal spot intensity of $\sim 10^{13} \mathrm{~W} \mathrm{~cm}^{-2}$ (Table 2). The angle between drive laser arms and XFEL probe was $22^{\circ}$. An ablation-driven compression wave was launched parallel to the sample normal over a $15.0 \mathrm{~ns}$ profile from a frequency doubled Nd:Glass laser system $(\lambda=527 \mathrm{~nm})$. By adjusting a waveplate optic on the long pulse laser, we could increase/decrease the total number of Joules in the drive pulse and achieve a range of pressures. The applied loading scheme is reverberation compression and was designed to achieve peak pressure and temperature in the water layer near the 7-9 ns X-ray probe time. The temporal drive profile was achieved by temporally advancing one of two laser beams. The first pulse, 10 ns duration, characterized by a $\sim 1.6 \times 10^{13} \mathrm{~W} \mathrm{~cm}^{-2}$ intensity, pseudo flat-top profile (Fig. 6, blue curve designated the AB Arm) was followed by a second pulse, after 5 ns. This second pulse was slightly less intense, $10 \mathrm{~ns}$ duration at $1.4 \times 10^{13} \mathrm{~W} \mathrm{~cm}^{-2}$ intensity, pseudo flat-top profile (Fig. 6 , green curve designated EF Arm). The target was exposed to the sum of these pulses in time-looking like a step shape in irradiance after $5 \mathrm{~ns}$. The optical laser and X-ray beam were spatially overlapped and operated in single shot mode. The absolute time zero corresponds to overlap of their leading edges. For each shot, a time delay was selected for the XFEL pulse relative to the optical laser pulse with a jitter of $0.35 \mathrm{~ns}$. XRD pattern was captured by CSPADs constructed of individual application-specific integrated circuits (ASICs).

The VISAR diagnostic resolves the velocity histories determined from a phase map of the data. Etalon thicknesses of $25.001 \mathrm{~mm}$ and $11.006 \mathrm{~mm}$ for Mach-Zehnder interferometer beds 1 and 2, respectively, enable a comparison of the two different velocity-time profiles. The profile match, and unique Up determination, is obtained from the correct number of $2 \pi$ fringe jumps allowed by the etalons. The target package (diamond-water-diamond) combined with this temporal drive profile were designed to generate the following sequence of events. First, the $\mathrm{AB}$ arm is incident on the diamond ablator and begins the ablation process setting up a shock wave in the ablator diamond. The impedance mismatch sets up a reverberating shock in the diamond ablator. Then, a weak shock traverses the water to $\sim 25 \mathrm{GPa}$ on the principal Hugoniot as transmitted by the elastic wave in diamond. The diamond plastic wave overtakes the weak elastic wave in the water and is the main compressive wave in the water reaching the diamond VISAR window at $\sim 5 \mathrm{~ns}$. This plastic wave sends a reverberation wave back into the water/ice again due to impedance mismatch. At $5 \mathrm{~ns}$, the second laser fires, $\mathrm{EF}$ arm, attempting to support continued reverberations.

Received: 23 April 2021; Accepted: 22 December 2021

Published online: 13 January 2022

\section{References}

1. Borucki, W. et al. Characteristics of planetary candidates observed by Kepler II. Analysis of the first four months of data. Astrophys. J. 736, 19 (2011).

2. Connerney, J., Acuna, M. \& Ness, N. The magnetic field of Neptune. J. Geophys. Res. 96, 19023-19042 (1991).

3. Stanley, S. \& Bloxham, J. Convective-region geometry as the cause of Uranus' and Neptune's unusual magnetic fields. Nature 428, 151-153 (2004).

4. Stanley, S. \& Bloxham, J. Numerical dynamo models of Uranus' and Neptune's magnetic fields. Icarus 184, 556-572 (2006).

5. Hubbard, W., Podolak, M. \& Stevenson, D. Interior of Neptune. In Interior of Neptune and Triton (ed. Cruisshank, D.) 109-138 (University of Arizona Press, 1995).

6. Redmer, R., Mattsson, T., Nettelmann, N. \& French, M. The phase diagram of water and the magnetic fields of Uranus and Neptune. Icarus 211, 798-803 (2011)

7. Celliers, P. et al. Electronic conduction in shock-compressed water. Phys. Plasmas 11, L41-L44 (2004).

8. Goldman, N., Fried, L., Kuo, I.-F. \& Mundy, C. Bonding in the superionic phase of water. Phys. Rev. Lett. 94, 217801 (2005).

9. Wilson, H., Wong, M. \& Militzer, B. Superionic to superionic phase change in water: Consequences for the interiors of Uranus and Neptune. Phys. Rev. Lett. 110, 151102 (2013).

10. Sun, J., Clark, B., Torquato, S. \& Car, R. The phase diagram of high-pressure superionic ice. Nat. Commun. 6, 8156 (2015).

11. Hernandez, A. \& Caracas, R. Superionic-superionic phase transitions in body-centered cubic H2O ice. Phys. Rev. Lett. 117, 135503 (2016).

12. Benoit, M., Bernasconi, M., Focher, P. \& Parrinello, M. New high-pressure phase of ice. Phys. Rev. Lett. 76, 2934 (1996).

13. Millot, M. et al. Experimental evidence for superionic water ice using shock compression. Nat. Phys. 14, 297-302 (2018).

14. Millot, M. et al. Nanosecond X-ray diffraction of shock-compressed superionic water ice. Nature 569, 251-255 (2019).

15. Gleason, A. et al. Compression freezing kinetics of water to ice VII. Phys. Rev. Lett. 119, 025701 (2017).

16. Wojdyr, M. Fityk: A general-purpose peak fitting program. J. Appl. Cryst. 43, 1126-1128 (2010).

17. Fei, Y. et al. Toward an internally consistent pressure scale. Proc. Natl. Acad. Sci. USA 104, 9182-9185 (2007)

18. Marsh, S. LASL Shock Hugoniot Data (University of California Press, 1980).

19. McWilliams, R. et al. Strength effects in diamond under shock compression to 0.1 to 1 TPa. Phys. Rev. B 81, 014111 (2010).

20. Knudson, M., Desjarlais, M. \& Dolan, D. Shock-wave exploration of the high-pressure phases of carbon. Science 322, $1822-1825$ (2008). 
21. Lee, K. et al. Laser-driven shock experiments on precompressed water: Implications for 'icy' giant planets. J. Chem Phys. 125, 014701 (2006).

22. Chau, R., Mitchell, A., Minich, R. \& Nellis, W. Electrical conductivity of water compressed dynamically to pressures of 70-180 GPa (0.7-1.8 Mbar). J. Chem. Phys. 114, 1361-1365 (2001).

23. Ree, F. Molecular interaction of dense water at high temperature. J. Chem. Phys. 76, 6287 (1982).

24. French, M., Mattsson, T., Nettelmann, N. \& Redmer, R. Equation of state and phase diagram of water at ultrahigh pressures as in planetary interiors. Phys. Rev. B 79, 054107 (2009).

25. French, M., Desjarlais, M. \& Redmer, R. Ab initio calculation of thermodynamic potentials and entropies for superionic water. Phys. Rev. E 93, 022140 (2016).

26. Lyon, S \& Johnson, J. SESAME database: Los Alamos Nat. Lab. LA-UR-92-3407 (1992).

27. Gleason, A. et al. Ultrafast visualization of crystallization and grain growth in shock-compressed SiO2. Nat. Commun. 6, 8191 (2015).

28. Dolan, D., Johnson, J. \& Gupta, Y. Nanosecond freezing of water under multiple shock wave compression: Continuum modeling and wave profile measurements. J. Chem. Phys. 123, 064702 (2005).

29. Celliers, P. et al. Line-imaging velocimeter for shock diagnostics at the Omega laser facility. Rev. Sci. Intrum. 75, 4916-4929 (2004).

30. Benedict, L. et al. Multiphase equation of state for carbon addressing high pressures and temperatures. Phys. Rev. B 89, 224109 (2014).

31. Larsen, J. \& Lane, S. Hyades: A plasma hydrodynamics code for dense plasma studies. J. Quant. Spectrosc. Radiat. Transfer 51, $179-186$ (1994).

32. Cascade Applied Sciences Inc. Hyades reference material: 'Material Constitutive Models' Chapter K.pdf (Cascade Applied Sciences Inc. (CAS), 2010).

33. Langford, J. \& Wilson, A. Scherrer after sixty years: A survey and some new results in the determination of crystallite size. J. Appl. Cryst. 11, 102-113 (1978).

34. Goncharov, A. et al. Dynamic ionization of water under extreme conditions. Phys. Rev. Lett. 94, 125508 (2005).

35. Sugimura, E. et al. Experimental evidence of superionic conduction in $\mathrm{H} 2 \mathrm{O}$ ice. J. Chem. Phys. 137, 194505 (2012).

36. Cavazzoni, C. et al. Superionic and metallic states of water and ammonia at giant planet conditions. Science 283, 44-46 (1999).

37. Mott, N. \& Gurney, R. Electronic Processes in Ionic Crystals 204 (Clarendon Press, 1940).

38. Karato, $\mathrm{S}$. Theory of isotope diffusion in a material with multiple species and its implications for hydrogen-enhanced electrical conductivity in olivine. Phys. Earth Planet. Int. 219, 49-54 (2013).

39. Nellis, W., Holmes, N., Mitchell, A., Hamilton, D. \& Nicol, M. Equation of state and electrical conductivity of 'synthetic Uranus', a mixture of water, ammonia, and isopropanol, at shock pressure up to $200 \mathrm{GPa}$ (2 Mbar). J. Chem. Phys. 107, 9096 (1997).

40. Loubeyre, P., LeToullec, R., Wolanin, E., Hanfland, M. \& Hausermann, D. Modulated phases and proton centering in ice observed by X-ray diffraction up to $170 \mathrm{GPa}$. Nature 397, 503-506 (1999).

41. Mattsson, T. \& Desjarlais, M. High energy-density water: Density functional theory calculations of structure and electrical conductivity. Sandia National Laboratory Report. SAND2006-7539 (2007).

\section{Acknowledgements}

AEG and WLM are supported by the Geophysics Program at NSF (EAR0738873) with additional support from LANL Reines LDRD and DOE FES ECA for AEG. BM received support from DOE award DE-SC0016248 and the University of California multi-campus research award 00013725. This work was performed at the Matter at Extreme Conditions (MEC) instrument of LCLS, supported by the U.S. DOE Office of Science, Fusion Energy Science under contract No. SF00515 (Grant Number: ECA 2019), and was supported by LCLS, a National User Facility operated by Stanford University on behalf of the U.S. DOE, Office of Basic Energy Sciences. We thank LCLS/SLAC staff for assistance during the experiment. Los Alamos National Laboratory is operated for the U.S. DOE National Nuclear Security Administration under contract DE-AC52-06NA25396.

\section{Author contributions}

C.B. participated in the experiment and developed XRD projection software. E.Ga., H.J.L., and E.Gr. participated in the experiment as MEC instrument scientists and contributed to data interpretation. P.C. and D.S. contributed to interpretation and provided VISAR modeling/interpretation. P.C. analyzed the VISAR traces. A.L., S.A., W.M., D.R., B.M., and S.S. contributed to data collection, analysis and/or interpretation/implications. A.G. was principal investigator and wrote the paper with contributions from co-authors.

\section{Competing interests}

The authors declare no competing interests.

\section{Additional information}

Correspondence and requests for materials should be addressed to A.E.G.

Reprints and permissions information is available at www.nature.com/reprints.

Publisher's note Springer Nature remains neutral with regard to jurisdictional claims in published maps and institutional affiliations.

Open Access This article is licensed under a Creative Commons Attribution 4.0 International License, which permits use, sharing, adaptation, distribution and reproduction in any medium or format, as long as you give appropriate credit to the original author(s) and the source, provide a link to the Creative Commons licence, and indicate if changes were made. The images or other third party material in this article are included in the article's Creative Commons licence, unless indicated otherwise in a credit line to the material. If material is not included in the article's Creative Commons licence and your intended use is not permitted by statutory regulation or exceeds the permitted use, you will need to obtain permission directly from the copyright holder. To view a copy of this licence, visit http://creativecommons.org/licenses/by/4.0/.

This is a U.S. Government work and not under copyright protection in the US; foreign copyright protection may apply 2022 